\title{
Current-induced torques in magnetic metals: Beyond spin-transfer
}

\author{
P.M. Haney ${ }^{\mathrm{a}, *}$, R.A. Duine ${ }^{\mathrm{b}}$, A.S. Núñez ${ }^{\mathrm{c}}$, A.H. MacDonald ${ }^{\mathrm{a}}$ \\ ${ }^{a}$ Department of Physics, The University of Texas at Austin, Austin, TX 78712-0264, USA \\ b Institute for Theoretical Physics, Utrecht University, Leuvenlaan 4, 3584 CE Utrecht, The Netherlands \\ ${ }^{\mathrm{c}}$ Departamento de Física, Universidad de Santiago de Chile, Casilla 307, Santiago, Chile
}

Available online 31 December 2007

\begin{abstract}
Current-induced torques (CITs) on ferromagnetic (FM) nanoparticles and on domain walls in FM nanowires are normally understood in terms of transfer of conserved spin angular momentum between spin-polarized currents and the magnetic condensate. In a series of recent articles, we have discussed a microscopic picture of CITs in which they are viewed as following from exchange fields produced by the misaligned spins of current carrying quasiparticles. This picture has the advantage that it can be applied to systems in which spin is not approximately conserved. More importantly, this point of view makes it clear that CITs can also act on the order parameter of an antiferromagnetic (AFM) metal, even though this quantity is not related to total spin. In this informal and intentionally provocative review we explain this picture and discuss its application to antiferromagnets.
\end{abstract}

(C) 2007 Elsevier B.V. All rights reserved.

Keywords: Spin torque; Antiferromagnets; Ferrimagnets

\section{Spin-transfer torques}

The study of spin-transfer torques began in 1996 when Slonczewski [1] and Berger [2] independently predicted that magnetization dynamics can be induced by current in circuits containing non-collinear magnetic elements. Berger's paper focused on the emission of spin waves as the source of magnetic excitations, while Slonczewski invoked conservation of spin angular momentum to infer magnetic dynamics. Slonczewski's observation that a net spincurrent flux into a volume of magnetic material implies that a torque acts on the magnetization in that volume is the key idea for most theories of current-induced torques (CITs). Over the past decade many experiments have confirmed [3-9] Slonczewski's predictions. There has also been theoretical progress, elaborating on Slonczewski's

\footnotetext{
${ }^{*}$ Corresponding author.

E-mail addresses: haney411@physics.utexas.edu, haney411@gmail.com (P.M. Haney), duine@phys.uu.nl (R.A. Duine), alvaro.s.nunez@gmail.com (A.S. Núñez), macd@physics.utexas.edu (A.H. MacDonald).

URLs: http://www.ph.utexas.edu/ ^haney411/paulh.html (P.M. Haney), http://www.phys.uu.nl/duine (R.A. Duine), http://www.ph.utexas.edu/macdgrp (A.H. MacDonald).
}

ideas and developing techniques which shed light on their implications for particular materials combinations and geometries. For example, Stiles and Zangwill explicitly exhibited all of the sources of net spin-current flux, namely spin-dependent transmission, spin precession, and rotation of reflected and transmitted spins [10]. Brataas et al. have formulated a general theory in which spin-dependent interface conductances, calculated from first principles or extracted from experimental data, can be combined using a generalized set of Kirchhoff laws to predict transport properties and magnetization dynamics in a circuit containing non-collinear magnetic elements [11]. Other approaches include solving generalized Boltzmann [12] or spin diffusion equations [13]. All of this theoretical work is directed towards evaluation of the net spin-current into a volume of material. Since the underlying systems possess conservation of total spin angular momentum, any theory for these systems must respect this global conservation law. For systems in which total spin is conserved, the relationship between net spin-current and torques is very general. Appealing to spin conservation enables reliable predictions to be made about current-induced magnetization dynamics without having to specify which spins form the macroscopic magnetization or the microscopic origin of the 
effective magnetic fields which cause them to precess. The conservation laws assure that if one does the "bookkeeping" of spin properly, the CIT acting on a volume may be inferred. We therefore sometimes refer to the point of view which utilizes powerful conservation-law consequences as the bookkeeping theory of spin-transfer. This point of view has had qualitative and quantitative success in describing experiments on spin-transfer in spin valve systems which are composed of several ferromagnetic (FM) nanoparticles, and on spin-transfer induced domain wall motion in FM nanowires.

The bookkeeping theory of spin-transfer torques raises two questions which often need not be answered explicitly and which initially drew our interest to the issue of CITs. In attempting to provide answers to these questions that we find satisfying, we have been led to the theoretical picture of CITs described below.

i. What is the distinction in the spin-transfer picture between the electrons that carry current and the electron spins that compose the magnetic condensate? This question is particularly relevant for the transition metal systems which are the workhorses of metal spintronics since we know that both s-like and d-like orbitals must be treated as itinerant. There is no clean distinction between the electrons which contribute the moments that order and the electrons that carry current.

ii. Does spin-transfer occur in systems with strong spin-orbit coupling? Because of spin-orbit coupling, spin angular momentum is never really conserved, even in perfect crystals that have no disorder. How strong does spin-orbit coupling need to be to weaken or eliminate the spin-transfer effect? This question is particularly relevant to FM semiconductors like $(\mathrm{Ga}, \mathrm{Mn}) \mathrm{As}$ in which the spin-orbit coupling strength is [14] comparable to the magnetic exchange energy and to the Fermi energy.

\section{CITs and non-equilibrium spin-densities}

In our picture spin-transfer torques arise as follows: The spin-density of electrons near the Fermi energy of a magnetic metal is altered when they carry a current through a non-collinear magnetic environment. The change occurs as they realign their spins to sample the exchange field of the ferromagnet and thereby steer their spin orientations to match their non-collinear environment. This change in spin-density leads to a change in the exchange field of the ferromagnet, which causes spins in orbitals far from the Fermi level to precess. We will refer to this as the CIT picture of spin-transfer. Although consistent with the bookkeeping theory of spin-transfer for systems in which total spin is conserved, it suggests that the phenomena is more general. One implication as we discuss below is that CITs drive order parameter dynamics in antiferromagnetic (AFM) metals.

\subsection{Current-induced torques}

We first introduce some notation and provide a general orientation. The formalism we describe is applicable to any mean field theory, but in this article we consider primarily systems described by the local spin-density approximation (LSDA) of density functional theory (DFT). The notation we use below is appropriate for a Hamiltonian in a tightbinding or local orbital basis. We separate both the single-particle Hamiltonian and the density matrix into spin-dependent and spin-independent contributions:

$\rho_{i^{\prime} i}=\frac{1}{2}\left[\rho_{i^{\prime} i}^{(0)}+\vec{m}_{i^{\prime} i} \cdot \vec{\tau}\right]$,
$H_{i^{\prime} i}=H_{i^{\prime} i}^{(0)}-\frac{1}{2} \vec{\Delta}_{i^{\prime} i} \cdot \vec{\tau}$,

where $\vec{\tau}$ is the vector of Pauli spin matrices and $i^{\prime}, i$ are site or orbital indices. In DFT, $H_{i^{\prime} i}$ is the Kohn-Sham singleparticle Hamiltonian. The notation for the spin-dependent part of the $H$ is chosen to emphasize that it produces a spin-splitting $\Delta$ when it is orbital independent, as is often assumed in simple toy models of an FM metal. (Note that $\vec{m}$ and $\vec{\Delta}$ are in general complex for orbital off-diagonal elements, i.e. for $i \neq i^{\prime}$; any Hermitian matrix may be uniquely written in the above form.) In LSDA, the interaction contribution to $\vec{\Delta}$ and $\vec{m}$ are related locally at each point in space according to

$\vec{\Delta}(\vec{r})=\Delta_{0}(n(\vec{r}), m(\vec{r})) \hat{m}$,

where $n$ and $\vec{m}=m \hat{m}$ are the local charge and spindensities, respectively, and $\Delta_{0}$ is some parameterization of the exchange-correlation potential. Note that $\vec{\Delta}(\vec{r})$ acts like an effective-magnetic field experienced by the Kohn-Sham quasiparticles. (In Eq. (2), $\vec{\Delta}(\vec{r})$ and $m(\vec{r})$ are functions in real space, while in Eq. (1), $\Delta_{i, i^{\prime}}$ represents the $i, i^{\prime}$ matrix element of the real space potential $\Delta(\vec{r})$, and $\vec{m}_{i, i^{\prime}}$ the spindependent part of density matrix in orbital space.) A local relationship in space does not imply proportionality of the orbital representation matrix elements. In particular, there are strong exchange interactions between s-like and d-like orbitals.

We define the difference between spin-densities in the presence and in the absence of a current as the nonequilibrium spin-density, denoted by $\vec{m}^{\mathrm{tr}}$, and the corresponding difference in exchange-correlation potentials is denoted by $\vec{\Delta}^{\text {tr }}=\Delta_{0}(n, m) \vec{m}^{\text {tr }} /|m|$. This expression assumes that $\left|\vec{m}^{\mathrm{tr}}\right| / m$ is small, something that is valid for any reasonable current strength. In a circuit with a noncollinear magnetic configuration, the contribution to the local exchange-correlation effective magnetic field from the non-equilibrium quasiparticles will in general point in a different direction than the magnetic condensate, and this misalignment is responsible for the ensuing torque on the magnetic condensate - the spin-transfer torque [15] (see Fig. 1). The non-equilibrium spin-density driven by a source-to-drain bias voltage in a specific nanoscale circuit can be evaluated theoretically using non-equilibrium Green's function (NEGF) techniques, as we describe 
below. For a bulk magnetic metal with a smooth spintexture, the non-equilibrium spin-density can be evaluated by treating both the spatial variation of magnetization direction and the electric field which drives a bulk current perturbatively.

As an example, consider the spin valve structure of two ferromagnets separated by a spacer. If a bias is applied, there is a component of the non-equilibrium spin-density which is perpendicular to the plane spanned by the two ferromagnets. Because this spin-density is not parallel to the exchange-correlation potential $\Delta$, the transport orbital spins precess as they move through the circuit to accommodate the change in exchange-field orientation. The magnetization of both layers will precess around the local out-of-plane exchange field generated by this nonequilibrium spin-density, and this precession is the one which leads to current-induced magnetization switching (CIMS) (see Fig. 2).

In the case where total spin is conserved, this view is essentially equivalent to the bookkeeping theory of spintransfer, as can be seen by evaluating $\mathrm{d} \vec{m}^{\text {tr }} / \mathrm{d} t$ for a currentcarrying quasiparticle with source to drain scattering boundary conditions:

$\frac{\mathrm{d} \vec{m}^{\operatorname{tr}}}{\mathrm{d} t}=\frac{1}{i \hbar}\left[\vec{m}^{\operatorname{tr}}, H\right]=\left(\vec{Q}_{1}-\vec{Q}_{n}\right)+\frac{1}{\hbar} \operatorname{Tr}\left[\operatorname{Re}\left[\vec{m}^{\operatorname{tr}} \times \vec{\Delta}\right]\right]$.

The above relation can be obtained by evaluating the commutator directly and using the commutation properties of Pauli spin matrices. From Eq. (1), $\vec{m}^{\text {tr }}$ is the spindependent part of the scattering state density matrix and $\vec{\Delta}$ is the spin-dependent part of the Hamiltonian, each of which is expanded in the three Cartesian components of Pauli matrices $\tau_{x}, \tau_{y}, \tau_{z}$. The trace in the second term is over orbitals in the subsystem (in the example shown in Fig. 2, the subsystem consists of all orbitals between planes 1 and $n$ ),

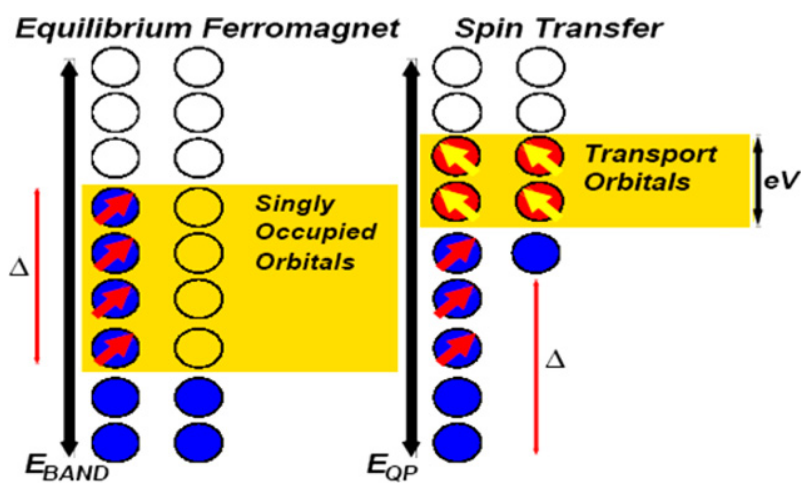

Fig. 1. Left panel: Ground state of a metallic ferromagnet. The lowenergy collective degree of freedom, the magnetization direction, is the spin orientation of singly occupied orbitals. Right panel: Quasiparticles experience a strong exchange field $\vec{\Delta}$ that brings majority and minority spins into equilibrium. Because this field is parallel to the magnetization it does not produce a torque. In an inhomogeneous ferromagnet, the spin orientation of the transport orbitals (in a window of width $\mathrm{eV}$ at the Fermi energy) must differ from the direction of the exchange-correlation potential $\Delta$. The current-transfer torque is then produced by the transport-orbital contribution to the exchange field.

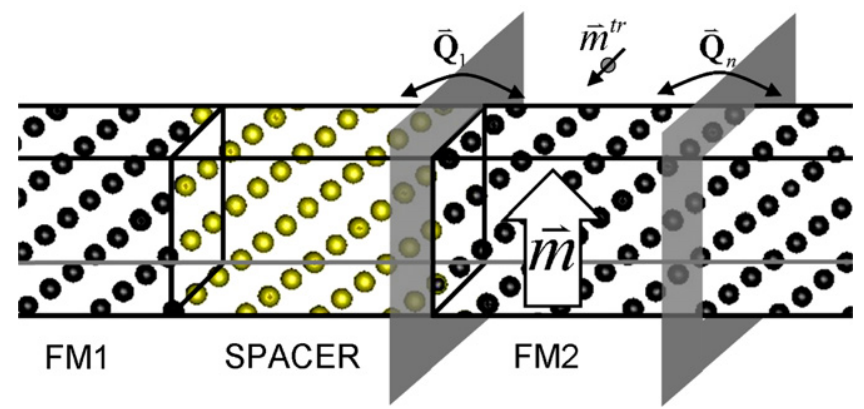

Fig. 2. Illustration of the relation between spin-current fluxes $\vec{Q}$ and nonequilibrium spin-densities $\vec{m}^{\mathrm{tr}}$. The planes through which spin-currents $\vec{Q}$ are evaluated are between layers labeled 0 and 1 , and $n-1$ and $n$.

and the spin-dependent part of matrices are multiplied in cross-product form.

The spin-current operator $\vec{Q}_{i}$ is defined as the spincurrent that flows between sites $i$ and $i+1$, and is given by

$\vec{Q}_{i}=\frac{1}{\hbar} \sum_{\substack{k \leq i \\ j>i}} \operatorname{Tr}\left[\vec{\tau}\left(\rho_{k j} H_{j k}-H_{k j} \rho_{j k}\right)\right]$.

In Eq. (4) the trace is over spin space, and $H_{i j}\left(\rho_{i j}\right)$ is the $2 \times 2$ spin matrix of the $i, j$-orbital component of $H(\rho)$. The second term of Eq. (3) represents the torque present on the quasiparticle due to its misalignment with the magnetic condensate. In steady state transport, the left-hand side of Eq. (3) vanishes, and so the divergence of the spincurrent-or the net spin-current flux, is equal to the quasiparticle-condensate CIT. The equation of motion for the magnetic condensate is [16]:

$\frac{\mathrm{d} \vec{m}}{\mathrm{~d} t}=-\vec{m} \times \vec{\Delta}=-\vec{m}^{\mathrm{tr}} \times \vec{\Delta}$.

From Eq. (3), this implies that the torque on $\vec{m}$ can be calculated with either $\vec{m}^{\text {tr }}$ (the non-equilibrium spindensity) or $\left(\vec{Q}_{1}-\vec{Q}_{n}\right)$ (the net spin-current flux), verifying the consistency between the approaches. In identifying the CITs as arising from non-equilibrium spin-densities, we have accomplished more than simply rephrasing the bookkeeping argument. We have identified the underlying microscopic interaction that is responsible for CITs. The same mechanism is operational in systems in which spin is not conserved. In addition, this microscopic view allows for the evaluation of local torques on individual atoms which can also drive the order parameter of antiferromagnets. The CIT which acts on the order parameter of a volume of AFM material is not related to the net spin-current flux into that volume. We comment more on these systems in Section 4.

\subsection{CITs and exchange interactions}

So far we have identified the CIT as resulting from the misalignment of the magnetic condensate with the exchange field contribution of non-equilibrium quasiparticles 
near the Fermi energy. This misalignment may seem out of place in view of Eq. (2) - in the ground state, the total spin and exchange field are aligned. However, even in equilibrium systems (systems that carry no charge current) with magnetic excitations (such as spin waves), the magnetic dynamics can be determined from an expression similar to Eq. (5) (assuming the excitation energy is "small" compared to other characteristic energies). In this case the Hamiltonian $H^{\prime}=H^{(0) \prime}-1 / 2\left(\vec{\Delta}^{\prime} \cdot \vec{\tau}\right)$ describing the magnetic excitation is constructed "by hand" starting from the ground state Hamiltonian (by, for example, applying a space-dependent spin rotation operator which describes a spin wave imposed on a collinear ground state Hamiltonian). The resulting non-self-consistent density matrix $\rho^{\prime}=$ $1 / 2\left(\rho^{(0) \prime}+\vec{m}^{\prime} \cdot \vec{\tau}\right)$ can then easily be calculated. The torque on the magnetic system is then given by $\operatorname{Tr}\left[\operatorname{Re}\left[\vec{m}^{\prime} \times \vec{\Delta}^{\prime}\right]\right]$, as in Eq. (5), and yields proper values for magnetic exchange energies and spin wave dispersion relations. Indeed, it can be shown that this approach to calculating properties of magnetic excitations is equivalent to previous approaches [17] which calculate $\delta E / \delta \hat{m}$ - the change inenergy associated with magnetic excitations $\delta \hat{m}$ [18]. (As a technical note, we remark that the evaluation of $\delta E / \delta \hat{m}$, as well as our evaluation of torques, relies on an adiabatic condition for magnetic dynamics. The adiabatic approximation follows from the fact that electronic time scales are much faster than the time scales which characterize collective magnetic dynamics [16]). Having established the close relationship between torques and variations in energy for excitations of equilibrium systems, one is naturally led to ask if such a relation exists for non-equilibrium systems. We address that point in Section 3.

\subsection{How to calculate non-equilibrium spin-densities}

We now briefly describe the technique we use to evaluate spin-densities and provide some references. This in some sense represents a technical point outside the focus of this article (although certainly a point of practical importance). We adopt a Landauer-Buttiker approach for describing non-equilibrium transport. In this approach, the bias voltage is represented by placing the system in contact with particle reservoirs with chemical potentials $\mu_{\mathrm{S}}=\varepsilon_{\mathrm{F}}+$ $e V_{\mathrm{B}} / 2$ and $\mu_{\mathrm{D}}=\varepsilon_{\mathrm{F}}-e V_{\mathrm{B}} / 2$ in source and drain, respectively. When the system Hamiltonian and its coupling to source and drain electrodes is time-independent (an assumption which follows from the adiabatic approximation described above), electrons with energies inside of the transport window $\mu_{\mathrm{D}}<E<\mu_{\mathrm{S}}$ solve a time-independent Schrodinger equation with incident-from-source scattering boundary conditions. In the Landauer-Buttiker approach, the conductance is simply proportional to the quantummechanical transmission probability of the scattering state. There are a number of techniques available to solve this system. We employ an NEGF method [19] combined with DFT [20]. The NEGF formalism yields all quantities of interest: the conductance, and the contribution to the density matrix from the non-equilibrium scattering states, from which $\vec{m}^{\text {tr }}$ is determined. A useful introduction to the NEGF formalism can be found in Ref. [21], and a more formal account is given in Ref. [22]. More details of how NEGF can be used to find CITs can be found in Ref. [23].

\section{Reactive and dissipative torques}

In the previous section we described how CITs arise as a consequence of the interaction between the magnetic condensate and the spin-density of non-equilibrium quasiparticles, a point of view that is an extension of the notion of spin-torques arising from spin-currents. The exchangecorrelation field of a non-self-consistent spin-density may also be associated with the torques arising from magnetic stiffness, and in this context these torques are related to the variation of an energy functional. We now comment on the possibility of finding CITs from an energy functional, which is of particular relevance in view of the ongoing discussion regarding the form of magnetization damping $[24,25]$.

The Landau-Lifshitz-Gilbert equation describes the dynamics of the magnetization. When spin-transfer torques are included, it is

$$
\frac{\partial \hat{m}}{\partial t}=\hat{m} \times\left(-\frac{\delta E[\hat{m}]}{\hbar \delta \hat{m}}\right)-\alpha \hat{m} \times \frac{\partial \hat{m}}{\partial t}+\left.\frac{\partial \hat{m}}{\partial t}\right|_{\mathrm{STT}} .
$$

Here $\hat{m}$ is the magnetization vector normalized to unit magnitude. The first term on the right-hand side describes precession of the magnetization around an effective field, written here as the functional derivative ${ }^{1}$ of the energy. In the so-called micromagnetic theory used to describe smooth spin-textures, the energy functional contains contributions from Zeeman coupling of the magnetization to an external magnetic field, anisotropy energy contributions due to spin-orbit coupling and magnetostatic interactions which violate spin conservation, as well as the energy cost of magnetization variation which is referred to in the context of micromagnetic theory as the exchange energy. (As experts are well aware, the theory of magnetism refers to many different things as exchange energies. The many uses of this word is fitting, since magnetism is always intimately related to electronic exchange processes, but it can be confusing.) In Eq. (6), the term proportional to $\alpha$ is the Gilbert damping term.

For smooth spin-textures, the spin-transfer torque terms can be expressed in terms of the leading order of a gradient

\footnotetext{
${ }^{1}$ The functional derivative $\delta E[\hat{m}] /(\delta \hat{m})$ is defined by the energy variation $\delta E=\int d \mathbf{x}[\delta E /(\delta \hat{m}(\mathbf{x}))] \cdot \delta \hat{m}(\mathbf{x})$ of the energy functional. Writing the energy in terms of an energy density $\mathscr{E}(\hat{m})$, i.e., $E[\hat{m}]=\int d \mathbf{x} \mathscr{E}(\hat{m}(\mathbf{x}))$, we have that

$\frac{\delta E[\hat{m}]}{\delta \hat{m}}=\frac{\partial \mathscr{E}}{\partial \hat{m}}-\frac{\partial}{\partial x_{\gamma}} \frac{\partial \mathscr{E}}{\partial\left(\partial \hat{m} / \partial x_{\gamma}\right)}$,

where a sum over the repeated index $\gamma \in\{x, y, z\}$ is again implied. See also the introductory article for a discussion of functional derivatives.
} 
(spatial derivative) expansion:

$$
\left.\frac{\partial \hat{m}}{\partial t}\right|_{\mathrm{STT}}=-\left(\mathbf{v}_{\mathrm{s}} \cdot \nabla\right) \hat{m}+\beta \hat{m} \times\left(\mathbf{v}_{\mathrm{s}} \cdot \nabla\right) \hat{m}
$$

where the velocity $\mathbf{v}_{\mathrm{s}}=-a^{3} P \mathbf{j} /|e|$ is proportional to the electric current density $\mathbf{j}$. Here $a$ is introduced as the lattice constant of a fictional lattice of unit magnetic moments representing a magnetization density $a^{-3}$, and $P$ is the spin polarization fraction of the current. Finally, the electron charge is denoted by $-|e|$. The first term in Eq. (7) is known as the adiabatic spin-torque. When $\mathbf{v}_{\mathrm{s}}$ is defined as above, this term is just the gradient expansion limit of the spinconserving spin-transfer torque discussed in the previous section. The second term proportional to the dimensionless parameter $\beta$ is commonly (and perhaps inappropriatelysee below) referred to as the non-adiabatic spin-torque. Since $\hat{m}$ is a unit vector, it is perpendicular to its time derivative $\dot{\hat{m}}$. It follows that when both $P$ and $\beta$ are treated as phenomenological parameters, Eq. (7) is quite general, assuming only that the CIT is linear in current. From a symmetry point of view, the first spatial derivative spintransfer torque terms in the generalized Landau-Lifshitz equations are allowed because current-flow breaks inversion symmetry. The micromagnetic exchange term, which is proportional to the second spatial derivative of the magnetization, is the leading order term in a gradient expansion in the absence of current. Eq. (7) can be derived microscopically [26-29], including the spin-transfer torque terms, by using non-linear response theory to describe the response of magnetization to external fields in the presence of a transport current.

The analogous equation for the free nanomagnet in a spin valve system is

$\left.\frac{\partial \hat{m}}{\partial t}\right|_{\mathrm{STT}}=-g j \hat{m} \times\left(\hat{m}_{\mathrm{p}} \times \hat{m}\right)-\beta g j\left(\hat{m}_{\mathrm{p}} \times \hat{m}\right)$,

with $\hat{m}_{\mathrm{p}}$ the magnetization direction of the pinned magnet. In this equation $g \sim a^{3} P /(2|e| \ell)$ with $\ell$ the length of the free ferromagnet in the direction of current flow. In this section we follow one common usage by referring to the first term as the Slonczewski spin-torque, and to the second term as the effective-field spin-torque; physically $g$ represents the efficiency of the Slonczewski spin-torque. An important observation is that Eqs. (7) and (8) are two sides of the same coin. If we put $\hat{m}_{\mathrm{p}}=\hat{m}(r-\mathrm{d} r)$ in Eq. (8), with $r$ the coordinate in the direction of the current, we obtain, after expanding to lowest order in $\mathrm{d} r$ the result in Eq. (7). In the case of spin valve structures we know from microscopic theory that the approximate expression for $g$ has corrections from spin-dependent reflection and other effects that are easily captured by microscopic interface calculations $[10,11]$.

A useful classification of the terms in Eq. (6) follows from an examination of how they behave under time reversal operations. Letting $t \mapsto-t$, we have that $\hat{m}, \hat{m}_{\mathrm{p}} \mapsto-\hat{m},-\hat{m}_{\mathrm{p}}$, and $\quad \mathbf{v}_{\mathrm{s}}, j \mapsto-\mathbf{v}_{\mathrm{s}},-j$. Furthermore, $E[-\hat{m}]=E[\hat{m}]$. After implementing this operation in
Eq. (6) we observe that there are two kinds of terms, reactive terms that are even under time reversal and dissipative terms that are odd. (Readers should be aware that there exist different points of view regarding this issue, found in Refs. [30] and [25].) The terms reactive and dissipative originate from linear response theory in which they play a similar role, distinguishing response that is in phase with a periodic driving force from response that is out of phase and therefore dissipative. The first and second terms on the right-hand side of Eqs. (7) and (8), respectively, represent corrections to the reactive and dissipative terms in Eq. (6) due to current flow. In the smooth texture (continuum) limit this is obvious from a microscopic point of view, because the terms proportional to $\alpha$ and $\beta$ emerge from microscopic linear-response theory as dissipative parts of the spin-density, spin-density response function in the presence of current [27]. To many readers the classification of the Slonczewski spin-torque as reactive may come as a surprise: after all, the Slonczewski spintorque competes with the Gilbert damping in currentdriven magnetization reversal. The latter phenomenon is dynamic, however, and does not imply that the Slonczewski spin-torque is dissipative. This classification is consistent with our picture that CIT phenomena primarily reflect precession around a transport-induced contribution to the exchange-correlation effective magnetic field.

The fact that both the adiabatic spin-torque and the Slonczewski spin-torque are both reactive, triggers the question whether they can be derived from an energy functional. Indeed, as we show below, the action

$$
\begin{aligned}
\mathscr{A}[\hat{m}]= & \int \mathrm{d} t\left\{-\left[\int \frac{\mathrm{d} \mathbf{x}}{a^{3}} \hbar v_{s, \gamma} A_{\gamma^{\prime}}(\hat{m}(\mathbf{x}, t)) \nabla_{\gamma} m_{\gamma^{\prime}}(\mathbf{x}, t)\right.\right. \\
& \left.\left.+\hbar \mathbf{A}(\hat{m}(\mathbf{x}, t)) \cdot \frac{\partial \hat{m}(\mathbf{x}, t)}{\partial t}\right]-E[\hat{m}]\right\},
\end{aligned}
$$

with A the vector potential of a magnetic monopole determined by

$\varepsilon_{\gamma \gamma^{\prime} \gamma^{\prime \prime}} \frac{\partial A_{\gamma^{\prime \prime}}}{\partial \hat{m}_{\gamma^{\prime}}}=\hat{m}_{\gamma}$

that enforces spin quantization [31], reproduces, upon variation, the equation of motion in Eq. (7) for $\alpha=\beta=0$ [32]. We note that in the above equation $\varepsilon_{\gamma \gamma^{\prime} \gamma^{\prime \prime}}$ is the antisymmetric Levi-Civita tensor and sums over repeated Cartesian indices $\gamma, \gamma^{\prime}, \gamma^{\prime \prime} \in\{x, y, z\}$ are implied.

To obtain the equation of motion corresponding to the action in Eq. (9) we have to calculate the variation of the action, i.e., $\delta \mathscr{A} / \delta \hat{m}_{\gamma}$ and put it equal to zero. We find in first instance that

$0=\frac{\delta \mathscr{A}}{\delta \hat{m}_{\gamma}}=\hbar\left(\frac{\partial A_{\gamma}}{\partial \hat{m}_{\gamma^{\prime}}}-\frac{\partial A_{\gamma^{\prime}}}{\partial \hat{m}_{\gamma}}\right)\left(\frac{\partial}{\partial t}+v_{s, \gamma^{\prime \prime}} \frac{\partial}{\partial x_{\gamma^{\prime \prime}}}\right) \hat{m}_{\gamma^{\prime}}-\frac{\delta E}{\delta \hat{m}_{\gamma}}$.

Using Eq. (10) we have that

$\frac{\partial A_{\gamma}}{\partial \hat{m}_{\gamma^{\prime}}}-\frac{\partial A_{\gamma^{\prime}}}{\partial \hat{m}_{\gamma}}=-\varepsilon_{\gamma^{\prime} \gamma^{\prime}} \hat{m}_{\gamma^{\prime \prime}}$, 
which we use to rewrite Eq. (11) as

$\hbar \hat{m} \times\left[\frac{\partial}{\partial t}+\left(\mathbf{v}_{\mathrm{s}} \cdot \nabla\right)\right] \hat{m}=\frac{\delta E}{\delta \hat{m}}$.

Taking the cross-product of the above equation with $\hat{m}$ and using the fact that $\hat{m}$ is a unit vector we obtain Eqs. (6) and (7) for $\alpha=\beta=0$.

The term in the action in Eq. (9) that is proportional to $\mathbf{v}_{\mathrm{s}}$ is physically understood as the Berry phase acquired by the electrons as they drift through a non-collinear magnetization texture. Such Berry phases occur naturally in spin systems. To see this in more detail consider first the simple case of a spin $S$ in a time-dependent unit magnetic field $\hat{m}(t)$ with hamiltonian $\mathscr{H}=-\hat{m}(t) \cdot \hat{S}$. Suppose the magnetic field is varied very slowly from $\hat{m}\left(t_{\mathrm{i}}\right)$ to $\hat{m}\left(t_{\mathrm{f}}\right)$, with $\hat{m}\left(t_{\mathrm{f}}\right)=\hat{m}\left(t_{\mathrm{i}}\right)$, so that the system remains in its ground state. One can show [31] that the quantum mechanical wave function of the spin acquires a non-trivial phase factor $\mathrm{e}^{-\mathrm{i} S \Omega}$. Here, $\Omega$ is the area on the unit sphere enclosed by the path that the spin traces out as it adiabatically follows the magnetic field. Using Stokes' theorem we write this area as a line integral over the boundary of $\Omega$, that is,

$\Omega=\int_{\Omega} \hat{m} \cdot \mathrm{d} \hat{a} \equiv \int_{\Omega}\left[\nabla_{\hat{m}} \times \mathbf{A}(\hat{m})\right] \cdot \mathrm{d} \hat{a}=\int_{\partial \Omega} \mathbf{A}(\hat{m}) \cdot \mathrm{d} \ell$,

with the monopole vector potential $\mathbf{A}(\hat{m})$ determined by Eq. (10). Note that the above also shows that although the vector potential is only defined up to a gauge transformation $\mathbf{A} \rightarrow \mathbf{A}-\nabla_{\hat{m}} \Lambda$, with $\Lambda$ an arbitrary function of $\hat{m}$, the physical quantity of interest, namely the area $\Omega$, is unaffected and well defined. Using the above results we observe that the term proportional to $\mathbf{v}_{s}$ in the action in Eq. (9) is determined by the area that the magnetization traces out on the unit sphere.

Having found an action that reproduces the equation of motion for the magnetization including the adiabatic spintransfer torque, we define an energy functional

$E_{j}[\hat{m}]=\int \frac{\mathrm{d} \mathbf{x}}{a^{3}} \hbar v_{s, \gamma} A_{\gamma^{\prime}}(\hat{m}(\mathbf{x}, t)) \nabla_{\gamma} m_{\gamma^{\prime}}(\mathbf{x}, t)+E[\hat{m}]$.

Using this energy functional the equation of motion for the magnetization direction is written as

$\frac{\partial \hat{m}}{\partial t}=\hat{m} \times\left(-\frac{\delta E_{j}[\hat{m}]}{\hbar \delta \hat{m}}\right)$.

To illustrate that the energy functional in Eq. (15) is indeed the energy that is minimized in the presence of current and as such a useful concept, we now add dissipative terms to the above equation. In particular, we consider the case $\alpha \neq 0$ and $\beta=0$. In that case we have that

$\frac{\partial \hat{m}}{\partial t}=\hat{m} \times\left(-\frac{\delta E_{j}[\hat{m}]}{\hbar \delta \hat{m}}\right)-\alpha \hat{m} \times \frac{\partial \hat{m}}{\partial t}$,

so that, since $\alpha>0$, the energy functional $E_{j}[\hat{m}]$ is indeed minimized according to the above equation of motion. This energy functional is relevant for understanding the socalled "intrinsic pinning" of a domain wall that occurs for $\beta=0$. "Intrinsic pinning" refers to the fact that a magnetic domain wall in a perfect material is displaced only for a sufficiently large applied current, and is "pinned" otherwise [33]. Minimization of this energy functional yields a physically clear explanation of intrinsic domain wall pinning $[33,34]$.

We end this section with some comments regarding spin valves. Writing down an action that reproduces Slonczewski's spin-torque directly for the spin valve case turns out to be more complicated because of the "discretization limit" one has to take in going from a smooth magnetization texture to a spin valve. We speculate, nonetheless, that the above action in principle is sufficient, as it reproduces Eq. (7) and we have seen the equivalence of Eqs. (7) and (8). At this point some readers may object that it is in fact the dissipative effective field torque proportional to $\beta$ in Eq. (8) which can be derived from the energy functional

$\tilde{E}[\hat{m}]=-\beta g j \hat{m}_{\mathrm{p}} \cdot \hat{m}$.

We note, however, that this energy is odd under time reversal and therefore not a proper energy. Only when the pinned magnet is kept fixed as $t \mapsto-t$ it is even under time reversal. This corresponds to regarding the pinned magnet as distinct from the dynamical system at hand (the free magnet).

Among the many questions which remain we mention two. (i) Is the energy functional from which the reactive spintorques are derived a practical concept? The prefactor of the monopole vector potential contains the spin-current evaluated in the collinear situation. Is this useful for calculations of the Slonczewski spin-torque efficiency? We note that a different perspective on this issue can be found in Ref. [35]. (ii) Can useful predictive expressions be derived for the dissipative coefficients $\alpha$ and $\beta$ of real FM materials?

\section{CITs in Ferrimagnets}

For systems in which spin is approximately conserved, such as transition metals, the CIT picture is complementary to the standard bookkeeping argument of spintransfer, as described earlier. For systems in which spin is not conserved (i.e. systems with strong spin-orbit coupling), the bookkeeping argument is no longer valid, and the CIT picture must be adopted. Examples of such systems include diluted magnetic semiconductors (DMS) and rare-earth elements. The CIT picture has been previously employed for these systems, for example by Nguyen et al. who calculate the current-driven domain wall mobility in the DMS (Ga,Mn)As [36].

Recent experiments on ferrimagnets provide an interesting test of CIT theories. Jiang et al. consider magnetoresistance and CIMS in a system consisting of an FM CoFe fixed layer and a ferrimagnetic CoGd free layer [37]. In CoGd, the magnetization of the two sublattices Co and Gd point in opposite directions. For temperatures above the magnetic compensation temperature $T_{\mathrm{MC}}$, the net magnetization points in the same direction as the Co sublattice, 
while for temperatures below $T_{\mathrm{MC}}$, it points in the direction of the $\mathrm{Gd}$ sublattice. The net magnetization vanishes at $T=T_{\mathrm{MC}}$.

Transport is dominated by orbitals from the Co sublattice, so that the magnetoresistance probes the relative orientation of the Co sublattice with the FM pinned layer. Therefore, the magnetoresistance changes sign at $T=T_{\mathrm{MC}}$ (so that for $T<T_{\mathrm{MC}}$, the magnetoresistance is negative-in this regime the Co sublattice is antiparallel with the net magnetization).

The experimental result of key interest is that the sense of the CIMS changes sign not at $T=T_{\mathrm{MC}}$, but at a higher temperature. The interpretation provided for this experimental result was that at all temperatures, the CIMS is determined by the relative orientation of the total angular momentum of pinned layer and free layers. The angular momentum $\vec{L}$ and magnetization $\vec{M}$ of a sublattice are related by $\vec{L}=\vec{M} / \gamma$, where $\gamma=g \mu_{\mathrm{B}} / \hbar$ is the gyromagnetic ratio, whose $g$-value depends on the degree of spin-orbit coupling. Making the assumption that different $g$-factors can be assigned to the Co and Gd sublattices, we can see that $\mathrm{CoGd}$ has the curious property that in a certain temperature range, its magnetization $\vec{M}=\vec{M}_{\mathrm{Co}}+\vec{M}_{\mathrm{Gd}}$ and angular momentum $\vec{L}=\vec{M}_{\mathrm{Co}} / \gamma_{\mathrm{Co}}+\vec{M}_{\mathrm{Gd}} / \gamma_{\mathrm{Gd}}$ can have opposite signs.

At temperatures $T>T_{\mathrm{MC}}$, the magnetization and angular momentum are parallel, and are dictated by the Co sublattice, while at very low temperatures, the magnetization and angular momentum are also parallel and dictated by the Gd sublattice. In these regimes, let us suppose that a positive current leads to parallel alignment of the CoGd magnetization with the fixed layer magnetization. In the intermediate temperature range where $\vec{M}$ and $\vec{L}$ have opposite sign, so the interpretation goes, a positive current aligns the angular momentum $\vec{L}$ (not $\vec{M}$ ) with the fixed layer magnetization.

We believe that this is an important experimental result for understanding the basic nature of CITs. The interpretation provided by the experimentalists extrapolates the bookkeeping theory from one based on conservation of only spin angular momentum to conservation of total angular momentum. We do not believe that this extrapolation is well motivated. For one thing, orbital angular momentum is not even approximately conserved in a crystal. We do not believe that the temperature at which the sense of CIMS changes sign must equal the temperature at which the total angular momentum changes sign. It is true that because of spin-orbit interactions, ferromagnets do have an orbital angular momentum, but neither spin nor orbital angular momentum are conserved. Indeed, understanding the magnitude of the orbital contribution to the magnetization of metallic ferromagnets has proved to be a challenging problem for many-body theory [38].

Our microscopic picture of CITs provides some guidance on how to think about these interesting experiments. A systematic inclusion of orbital magnetism is provided by current-density functional theory (CDFT) $[39,40]$. CDFT is an extension of DFT in which the current density (as opposed to charge density) plays the central role, and is able to treat many-body systems in magnetic fields of arbitrary strength [40]. The exchange-correlation potential is more complicated in CDFT than in DFT, and includes a vector potential. However, a standard practice is to simply employ DFT with the standard exchange-correlation potentials that depend only on spin-density, even when spin-orbit coupling is included in the Kohn-Sham singleparticle equations. One formal justification for this comes from the relativistic spin-density-functional formalism [41]. This formalism allows for relativistic corrections to the exchange-correlation potential, but the corrections do not have an overwhelming effect. Taking this as a starting point, the current-induced spin-torques in CoGd would be proportional to induced spin-densities and would be influenced by the spin-orbit interaction terms in the Kohn-Sham equations for the current-carrying quasiparticles.

Another aspect that appears to play a crucially important role is the thermal fluctuations of the magnetization, something which has not usually been accounted for by theory. The effect of these fluctuations is not clear $a$ priori (and indeed represents an interesting avenue of research in its own right). At finite temperature, both the Gd moments and the Co moments will fluctuate in orientation, so that the magnetization cannot be assumed to be collinear even within the CoGd nanomagnet. Presumably the change in sign of the average magnetization occurs for $T>T_{\mathrm{MC}}$ because the Gd moment orientations fluctuate more strongly.

It is interesting to consider how we would expect thermal fluctuations in such a system to influence macroscopic CITs. Is the explanation for these experimental results related only to thermal fluctuations, with spin-orbit coupling playing an inessential role? Since the sum of the current-induced spin-torques on individual atoms will not in general be perpendicular to the total spin-density, its effectiveness in driving spatially coherent precession of the typical non-collinear spin-density is not simply related to the net spin-current through the nanomagnet. The intriguing experimental results in CoGd may indeed be pointing to a non-trivial role for thermal fluctuations of the spin-density in CIT phenomena, when these fluctuations are large.

\section{Spintronics in antiferromagnets}

The more general nature of the CIT picture suggests that it should be operational in more general circumstances, for examples in materials with more complex magnetic order than simple collinear ferromagnetism. We have recently considered [42-46] the effect of CITs in circuits containing different combinations of AFM and FM materials. To date, the role of antiferromagnets in spintronic devices is to pin an FM layer's orientation via an effect known as "exchange bias" [47,48]. We propose that AFM materials 
can serve as a building block for circuits which display effects such as giant magnetoresistance (GMR) and CIMS, with the staggered order parameter of the AFM playing the role of the orientation of the FM. We briefly discuss our results for the sake of illustrating the qualitatively new features than can emerge from AFM spintronics. These early efforts may help point the way to fruitful directions to consider in moving forward.

The property that the local spin-transfer torque on each atomic site is equal to the net spin-current into that site is equally true for ferromagnets and antiferromagnets. The difference between the two cases is the nature of the magnetic order. The magnitude of CITs is nearly always dwarfed by the size of the torques caused by equilibrium exchange interactions when the relative orientations of different moments is distorted. In the case of a ferromagnet, equilibrium exchange orientations keep all the moments in a nanomagnet essentially rigidly parallel; the CITs are generally not strong enough to change these relative orientations. The sum of all the CITs acts on the overall orientation of all the moments. For this mode of magnetic dynamics the CIT only has to compete with much weaker anisotropy, magnetostatic, external field, and damping effects. In an antiferromagnet, the mode of magnetic dynamics on which the CIT can have an effect is the rigid motion of all the moments of the antiferromagnet. For an antiferromagnet with two sublattices with opposite orientations, it is the difference between the sums of the torques over the two sublattices which has an effect.

GMR and CIT effects in ferromagnets rely on the interplay between electron transport and magnetic order. The source of this interplay is the strong spin-dependent exchange-correlation potentials seen by current-carrying quasiparticles, which is the result of a spin-dependent Fermi surface. Antiferromagnets do not posses a spindependent Fermi surface, the characteristic that is so essential to conventional spintronics, so it is clear that any GMR and CIT effects in AFM circuits must have a fundamentally different origin. (Indeed the only qualitative imprint of the ordered state on the electronic structure of an AFM is the formation of a gap at the spin-density wave vector; we denote the spin-density wave vector by $\vec{Q}$.) We can classify our studies by the relative orientation of the current $\vec{J}$ and the spin-density wave vector $\vec{Q}$.

Case 1: $\vec{J} \| \vec{Q}$. In Ref. [42] we considered an antiferromagnet in which the exchange splitting changed sign on alternate lattice sites in the current-motion direction $(\vec{J} \| \vec{Q})$. The structure we studied with this kind of model was similar to that of a normal spin valve, except that the magnetic nanoparticles separated by a normal metal spacer were AFM rather than FM. We refer to this type of structure as an AFM spin valve. The model we studied in this paper was a single-band toy model. In the $\vec{J} \| \vec{Q}$ case the interface layer of the AFM is uncompensated, i.e. it has non-vanishing total spin. In this model, we find a difference in conductivity according to whether or not the AFM layers adjacent to the spacer are parallel or anti-parallel, an effect we refer to as antiferromagnetic giant magnetoresistance (AGMR). We also find the remarkable property that when the AFM layers are non-collinear, the out-of-plane spin-density in the AFM is exactly constant in our lattice model antiferromagnet and exactly periodic in a continuum model antiferromagnet. Since the out-of-plane spin-density is responsible for the CIT, the torque is also constant in magnitude and alternating in sign throughout the volume of the AFM. By itself, this property implies that the critical current for switching is independent of the AFM layer thickness. This is in stark contrast to the FM case, where the spin-torque decays rapidly away from the interface as the result of interference between different transverse channels' spin-density.

Since the staggered magnetization of antiferromagnets is not conserved, the staggered torque which drives order parameter dynamics is not protected by robust conservation laws. Indeed, both the AGMR and the CITs of the uncompensated AFM spin valve toy model can be seen to follow from phase-coherence effects: specifically the difference in the phase acquired by up and down spins as they traverse the circuit in various transverse channels. The presence of the CIT in this geometry may also be understood from the bookkeeping perspective: the difference in phase acquired by the up and down-component of an electron spin as it traverses a single uncompensated layer results in a net spin flux into that layer.

The toy model calculations demonstrate that GMR can occur in purely AFM spin valves and that CITs can drive AFM order parameters. At the same time, the toy model considerations also suggest that in the AFM case the effects are more easily weakened by inelastic scattering and more sensitive to the details of the various interfaces in these layered systems [43].

The toy model does not fully capture all the physics that is present in realistic AFM spin valve structures. To partially assess the significance of real-world complications we have performed $a b$ initio calculations for a system consisting of AFM Cr leads separated by a Au spacer [44]. The AGMR in this calculation is not primarily due to phase-coherent effects, but rather to spin-polarized interface resonance. The [ $\left.\begin{array}{lll}0 & 1\end{array}\right]$ surface of $\mathrm{Cr}$ with spin density

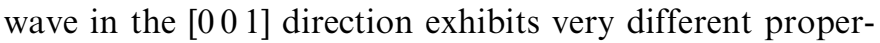
ties than that of the bulk, with an enhanced magnetic moment and a spin-dependent local density of states at the surface [49]. The spin-dependence of the surface density of states at the Fermi energy results in a spin-polarized current as electron flow passes through the surface. The presence of Au spacer has little effect on this surface state, and the Cr layer can essentially be thought of similarly as an FM layer, with the surface magnetization playing the role of the FM magnetization.

Another different aspect of this system is that the nonequilibrium out-of-plane spin-density is not periodic as in the simpler models, but partially decays away from the interface, because of the complex Fermi surface of Cr. For many transverse channels, the spin-dependent scattering 
state is a linear combination of Bloch states with different wave-vectors in the transport direction $k_{z}$. The spindensities of these scattering states then show an oscillatory spatial structure, and averaging over the Fermi surface results in destructive interference of the non-equilibrium spin-density away from the interface, as in the conventional FM case. We address some open questions regarding this system at the end of this section.

Case 2: $\vec{J} \perp \vec{Q}$. We have also considered a system consisting of a ferromagnet and antiferromagnet in which the current is perpendicular to the spin-density wave $(\vec{J} \perp \vec{Q})$. The total magnetization of each layer in the current-carrying direction vanishes in this case, i.e. the magnetization is compensated. Most AFM materials used in magnetoelectronics are fully compensated, i.e. the spindensity sums to zero in every lattice plane perpendicular to the current direction, or at least nearly so. Direct interfaces between nearly compensated antiferromagnets, which is perform the exchange bias function, and ferromagnets are common in spintronic circuits. The CITs discussed in this section can be dominant when the AFM and FM layers are separated by a spacer which reduces the importance of direct exchange interactions between the two actors. Symmetry considerations imply that the CITs between ferromagnets and compensated antiferromagnets differ qualitatively from the torques between ferromagnets.

The total CIT acting on an FM nanoparticle can always [10] be expressed in terms of the difference between incoming and outgoing spin-currents. The presence of a ferromagnet will in general induce a non-zero spin-current at the AFM-FM interface. When spin-polarized electron flux from the AFM with orientation $\hat{n}_{\mathrm{AFM}}$ enters an FM with orientation $\hat{n}_{\mathrm{FM}}$, the spin-current entering the FM will have some component in the $\hat{n}_{\mathrm{AFM}}$ direction. It follows that, just as in the familiar case where both materials are FMs, a CIT will act in the plane defined by $\hat{n}_{\mathrm{AFM}}$ and $\hat{n}_{\mathrm{FM}}$, as illustrated in Fig. 3. (Out of plane torques are also nonzero but tend to be much smaller.) Spin rotational invariance of the overall circuit implies that the in-plane torque must be an odd function of the angle $\theta$ between $\hat{n}_{\mathrm{FM}}$ and $\hat{n}_{\mathrm{AFM}}$ and that it can therefore be expanded in terms of a sine-only Fourier series, vanishing for both parallel and antiparallel collinear configurations. In this case, reversal
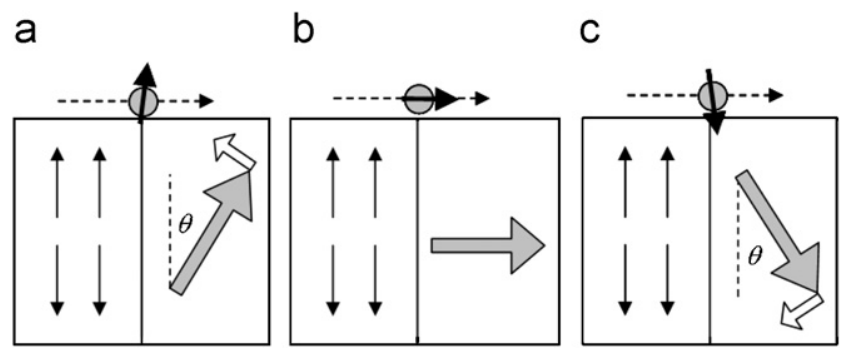

Fig. 3. CITs due to a compensated antiferromagnet. The arrows above the structure indicate the electron flux spin direction. The white arrows indicate the ensuing current-induced torques on the FM. The torque vanishes at $\theta=90^{\circ}$, and varies as $\sin 2 \theta$. of the AFM moment direction is equivalent to a lateral translation which cannot influence the CIT. It follows that in the compensated AFM case the torque is invariant under $\theta \rightarrow \theta+\pi$, restricting its Fourier expansion to terms proportional to $\sin (2 n \theta)$. It follows that the torque vanishes when $\hat{n}_{\mathrm{FM}}$ is perpendicular to $\hat{n}_{\mathrm{AFM}}$, and undergoes a sign change for $\theta \rightarrow \pi-\theta$, as illustrated in Fig. 3. The property that the torque acting on an FM due to a compensated AFM vanishes not only for collinear but also for perpendicular orientations is primarily responsible for a novel CIT phase diagram, which differs drastically from the now familiar applied-field/current phase diagram for FM layers [8].

We emphasize that in making this general argument of the symmetry-constrained form of the CIT on the FM, we have appealed to the bookkeeping picture. The bookkeeping picture combined with the assumption that the spin current becomes aligned to the local magnetization represents a simplification of great conceptual and practical utility. The calculation of the total CIT in a spin valve then requires only the determination of the spin-current in the spacer. On the other hand, for the AFM it is necessary to find the staggered torque, and this convenient picture of finding net torques from a single spin-current value does not apply. To date, we have found torques on AFMs by adding up torques on individual atoms, and their form and magnitude seemingly cannot be so easily be anticipated a priori.

We have performed a realistic calculation of the torques present when FM Co is adjacent to the AFM compound NiMn. As expected on the basis of the symmetry considerations explained above, the CIT is of $\sin 2 \theta$ form. We find that the CIT efficiency acting on both layers is substantial and of the same magnitude as that found in common FM systems. For electron flow from FM to AFM, the CIT tends to align the axis of the AFM with that of the FM, and to make the FM perpendicular to the axis of the AFM. Conversely, for electron flow from AFM to FM, the CIT tends to align the FM with the AFM axis, and make the AFM axis perpendicular to the FM (within their common plane). Put another way: the CIT tends to drive the orientation of the downstream material (AFM of FM) parallel with that of the upstream, and to drive the upstream material orientation perpendicular to the downstream.

The effect of such a torque on the stability of the FM orientation is most unusual for electron flow from FM to AFM. If the FM is an easy plane ferromagnet (hard axis $=\hat{x}$ ), and the $\mathrm{AFM}$ axis $(\mathrm{AFM}$ axis $=\hat{z})$ is assumed to lie in the ferromagnet's easy plane and be fixed, then for sufficiently large current, the stable configuration for the FM is to point approximately out of the easy plane. Fig. 4 shows the stable magnetization orientation phase diagram versus applied field and current. The applied field is scaled by the demagnetization field of the FM, $h=H_{\text {app }} / H_{\text {demag }}$, and the conversion of the dimensionless CIT $h_{\text {CI }}$ into a real current $J$ (assuming a demag field of 

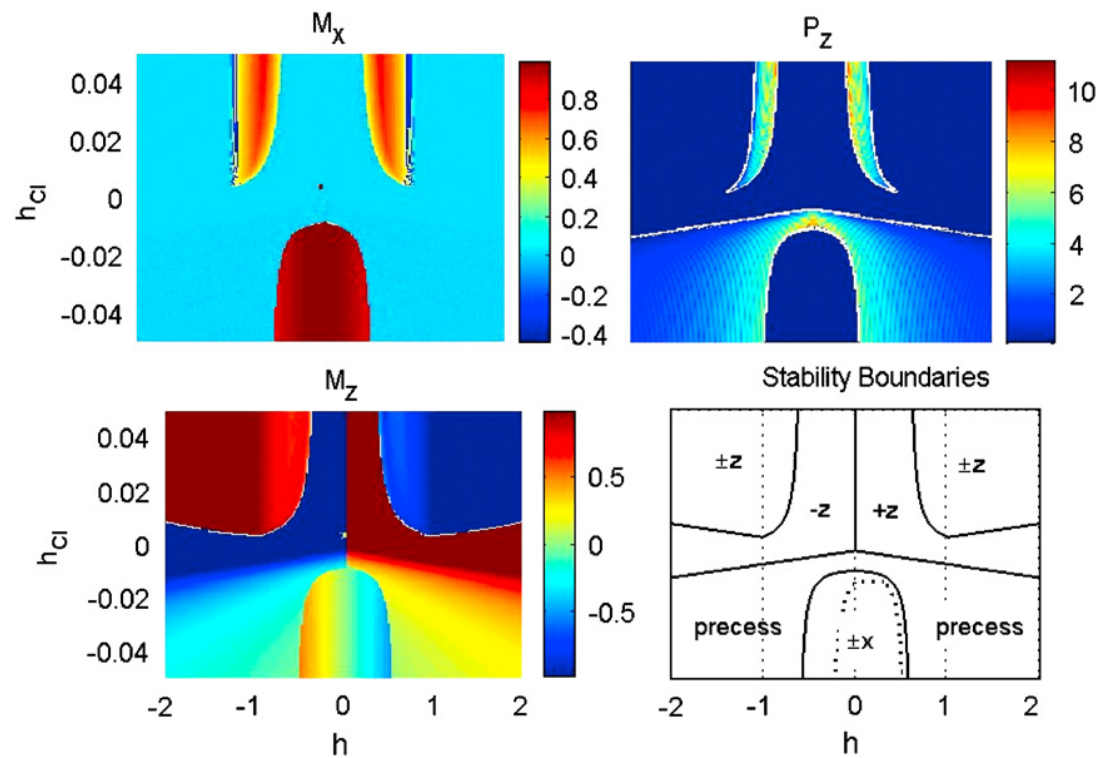

Fig. 4. Magnetic configuration $\left(M_{x}, M_{z}\right)$ and peak of power spectrum $P_{z}$ (arbitrary units) versus applied field and current. Also shown is the stability boundaries found analytically (the labels $\pm x, \pm z$ refer also to solutions which point approximately in these directions). The stability boundary plot also shows the reduced out-of-plane solution space for negative to positive field sweep with a dashed line.

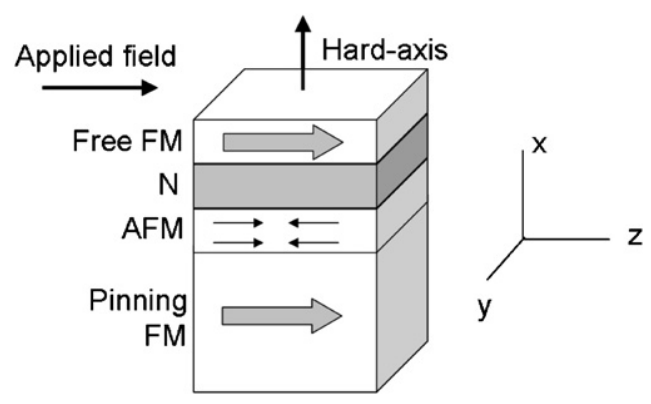

Fig. 5. Possible stack configuration to study the influence of compensated AFM on FM orientation as a function of applied field and current. The AFM is assumed to be fixed by the larger pinning FM via the exchange bias effect.

$1 \mathrm{~T})$ is $J=\left(h_{\mathrm{CI}} t\right) \times 3.8 \times 10^{9} \mathrm{~A} / \mathrm{cm}^{2}$, where $t$ is the thickness of the FM layer in $\mathrm{nm} ; h_{\mathrm{CI}}<0$ represents particle flow from FM to AFM.

A stack design which may be employed in future studies to investigate this effect is shown in Fig. 5. The AFM should be pinned in some way, here we have indicated pinning with the exchange bias effect by placing a larger FM adjacent to the AFM. As discussed in Ref. [45], it should not be necessary for the AFM to be single domain in order to see the effect. The orientation of the free FM may be detected via the GMR effect with the pinning FM. The pinning FM may play a role in the stability of the free FM, but its role is sufficiently distinct from that of the AFM that it may be possible to nevertheless detect the AFM CIT. A detailed discussion of this unexpected behavior can be found in Ref. [45]. The qualitatively distinct nature of the applied current-applied field phase diagram suggests that there may be other interesting aspects of this form of torque to be explored as well.
What next for AFM spintronics? There are other permutations of $\vec{J}, \vec{Q}$ orientation and FM/AFM stack design to be explored. We believe the key step necessary for making further progress is finding materials systems which clearly demonstrates the effect of CITs on or by an AFM layer in a way which can be distinguished from FM STT.

Experimental challenges abound for studying AFM spintronics. Chief among them is that the structure and orientation of an AFM is difficult to measure and difficult to control. The easiest way to control the AFM orientation is with an adjacent FM via the exchange bias effect. However this FM layer may exert spin-torques of its own, potentially obscuring the role of the AFM. It would be preferable therefore to avoid using a pinning FM, or to somehow remove its effect on transport. To the extent that effects rely on flat interfaces or phase-coherence, the experimental challenges become more severe. Nevertheless, recent experiments [46] established a dependence of unidirectional exchange bias fields on current, providing indirect evidence that CITs are present in AFMs. Other experiments attribute observed changes in exchange bias, steps in differential resistance, and the statistics of thermally activated switching to CITs on the antiferromagnet of an exchange-biased spin valve [50].

The role of disorder on both toy and more realistic models is likely much more important in antiferromagnets than in ferromagnets. This role has not yet been realistically assessed, and represents an important step forward in determining the viability of AFM spintronics which can be achieved by theoretical work alone. For example, some important properties of the $\vec{J} \| \vec{Q}$ geometry seem to rely to some extent on a clean, uncompensated AFM surface. The robustness of the GMR and CIT when 
this theoretical assumption is relaxed is still largely unknown.

Finally, so far we have only considered the effect of current on AFMs in multilayer geometries. It remains to study the effect of current on continuous AFM textures and domain walls, although some work is currently underway [51]. Domain walls in AFMs display interesting properties in their own right, including evidence of quantum tunnelling [52]. Clearly there is a wide range of phenomena in AFM spintronics that is yet to be explored.

\section{Spintronics in molecular systems/Pseudospintronics}

In the previous two sections, new and interesting physics was revealed by considering CIT effects in materials other than FM (ferrimagnets and antiferromagnets). That provokes the question of what other types of materials or systems might provide interesting manifestations of CIT.

One type of system in which CIT effects may occur in interesting ways is in molecular systems, or those with reduced dimensionality. So far experiments have focused on magnetoresistance effects for systems with FM leads sandwiching monolayers of molecules [53] or carbon nanotubes [54], for example. The NEGF is well suited for calculating spin-dependent transport for such systems [55]. The next step would be to consider the action of current on magnetic molecules to see if current-induced switching of the molecular spin is possible, and to consider experimental signatures of such a switching event [56,57]. Experimental challenges are abundant, and certainly outside of the expertise of these authors, however first-principles calculations may be useful in aiding the identification of the optimum experimental choice for molecule and lead material. There can however be difficulties in applying DFT (strictly speaking a ground state mean-field theory) directly to molecular transport (a non-ground state system in which electron correlations may be important) [58-60]. It is nevertheless likely that the current-induced torques in such systems will be qualitatively different than in conventional FMs. In FMs, the properties of CITs rely critically on the dimensionality (the spin-current becomes aligned to the local magnetization only after averaging over all incoming electron velocity directions), and these molecular systems are effectively $1-\mathrm{d}$ or $0-\mathrm{d}$.

Finally we mention that CITs are closely related to physics that occurs in other kinds of systems. For example, the supercurrent that flows through a superconductor in a circuit with a normal metal source and drain can be thought of as being driven by current-induced changes in the equation of motion for the order parameter. In this case, total particle number (a scalar) rather than total spin (a vector) is conserved. The case of circuits containing superconductors is therefore closely analogous to the case of $\mathrm{XY}$ easy-plane ferromagnets for which only the $\hat{z}$-spin is conserved. Bilayer quantum Hall systems near filling factor $v=1$, have an exciton condensate [61] ground state with spontaneous phase coherence in the two layers. The conserved quantity in this case is the difference between the numbers of particles in the two layers. The anomalous transport properties of these exciton superfluids are closely related [62] to the anomalous transport properties of magnetic systems that we have discussed in this review. We suspect that many other examples of current-driven order parameters will be discovered and exploited in the future. So far, metallic magnetism has provided the most phenomenologically rich and most extensively explored example of this type of physics. The lessons learned from this still developing body of research may have implications beyond the realm of magnetism.

\section{Conclusion}

In this review, we have described a microscopic theory of current-induced torques which identifies the interaction between misaligned spins of non-equilibrium quasiparticles and the magnetic condensate as the source of the torque. This perspective suggests that the phenomena of currentdriven order parameters is more general than the spintransfer idea. It suggests that in magnetic systems the phenomena is not limited to ferromagnets, or to systems in which total spin is conserved. We have applied this picture to consider spintronics in antiferromagnets, and have found a number of qualitative differences in the physics of CITs compared to the ferromagnetic case. There are a number of new areas that have not yet been fully explored with this approach - among them systems with strong spin-orbit coupling, different types of antiferromagnetic systems, and molecular systems. It is our hope that in exploring these novel systems, the key, fundamental aspects of CITs can be illuminated in their most general form.

\section{Acknowledgments}

We would like to acknowledge stimulating discussions with Hong Guo, Olle Heinonen, Enrico Rossi, Neal Smith, Maxim Tsoi, and Derek Waldron.

The work of R.A.D. is partially supported by the Stichting voor Fundamenteel Onderzoek der Materie (FOM) and the Nederlandse Organisatie voor Wetenschappelijk Onderzoek (NWO). The work of A.S.N. is partially supported by Proyecto Bicentenario de Ciencia y Tecnología ACT027. Work at UT Austin was supported in part by Seagate Corporation and by the National Science Foundation under Grant DMR-0606489.

\section{References}

[1] J. Slonczewski, J. Magn. Magn. Mater. 62 (1996) 123.

[2] L. Berger, Phys. Rev. B. 54 (1996) 9353.

[3] M. Tsoi, A.G.M. Jansen, J. Bass, W.C. Chiang, M. Seck, V. Tsoi, P. Wyder, Phys. Rev. Lett. 80 (1998) 4281.

[4] M. Tsoi, A.G.M. Jansen, J. Bass, W.C. Chiang, V. Tsoi, P. Wyder, Nature 406 (2000) 46

[5] J.A. Katine, F.J. Albert, R.A. Buhrman, E. B Myers, D.C. Ralph, Phys. Rev. Lett. 84 (2000) 3149. 
[6] F.J. Albert, N.C. Emley, E. B Myers, D.C. Ralph, R.A. Buhrman, Phys. Rev. Lett. 89 (2002) 226802.

[7] M.R. Pufall, W.H. Rippard, T.J. Silva, Appl. Phys. Lett. 83 (2003) 323.

[8] S.I. Kiselev, J.C. Sankey, I.N. Krivorotov, N.C. Emley, R.J. Schoelkopf, R.A. Buhrman, D.C. Ralph, Nature 425 (2002) 380.

[9] J. Grollier, V. Cros, H. Jaffres, A. Hamzic, J.M. George, G. Faini, J. Ben Youssef, H. Le Gall, A. Fert, Phys. Rev. B 67 (2003) 174402.

[10] M.D. Stiles, A. Zangwill, Phys. Rev. B 66 (2002) 014407.

[11] A. Brataas, G.E.W. Bauer, P.J. Kelly, Phys. Rep. 427 (2006) 157.

[12] J. Xiao, A. Zangwill, M.D. Stiles, Phys. Rev. B 70 (2004) 172405.

[13] A. Shpiro, P.M. Levy, S. Zhang, Phys. Rev. B 67 (2003) 104430.

[14] T. Jungwirth, J. Sinova, J. Masek, J. Kucera, A.H. MacDonald, Rev. Mod. Phys. 78 (2006) 809.

[15] A.S. Núñez, A.H. MacDonald, Solid State. Commun. 139 (2006) 31.

[16] V.P. Antropov, M.I. Katsnelson, B.N. Harmon, M. van Schilfgaarde, D. Kusnezov, Phys. Rev. B 54 (1996) 1019.

[17] A.I. Leichtenstein, M.I. Katsnelson, V.P. Antropov, V.A. Gubanov, J. Magn. Magn. Mater. 67 (1987) 65.

[18] P.M. Haney, Spintronics in Ferromagnets and Antiferromagnets From First Principles PhD Thesis, The University of Texas at Austin, 2007.

[19] S. Datta, Electronic Transport in Mesoscopic Systems, Cambridge University Press, Cambridge, 1995.

[20] J. Taylor, H. Guo, J. Wang, Phys. Rev. B 63 (2001) 245407.

[21] S. Datta, Superlattices Microstructures 28 (2000) 253.

[22] H. Haug, A.-P. Jauho, Quantum Kinetics in Transport and Optics of Semiconductors, Springer, Berlin, 1998.

[23] P.M. Haney, D. Waldron, R.A. Duine, A.S. Núñez, H. Guo, A.H. MacDonald, Phys. Rev. B 76 (2007) 024404.

[24] M.D. Stiles, W.M. Saslow, M.J. Donahue, A. Zangwill, Phys. Rev. B 75 (2007) 214423.

[25] N. Smith, arXiv:0706.1736, 2007.

[26] J. Fernández-Rossier, M. Braun, A.S. Núñez, A.H. MacDonald, Phys. Rev. B 69 (2004) 174412.

[27] R.A. Duine, A.S. Núñez, J. Sinova, A.H. MacDonald, Phys. Rev. B 75 (2007) 214420.

[28] H. Kohno, G. Tatara, J. Shibata, J. Phys. Soc. Jpn 75 (2006) 113706.

[29] Y. Tserkovnyak, H.J. Skadsem, A. Brataas, G.E.W. Bauer, Phys. Rev. B 74 (2006) 144405.

[30] W.M. Saslow, arXiv:0708.0997, 2007.

[31] A. Auerbach, Interacting Electrons and Quantum Magnetism, Springer, New York, 1994.

[32] Y.B. Bazaliy, B.A. Jones, S.-C. Zhang, Phys. Rev. B 57 (1998) R3213.

[33] G. Tatara, H. Kohno, Phys. Rev. Lett. 92 (2004) 086601.

[34] R.A. Duine, A.S. Núñez, A.H. MacDonald, Phys. Rev. Lett 98 (2007) 056605.
[35] M. Stamenova, S. Sanvito, T.N. Todorov, Phys. Rev. B 72 (2005) 134407.

[36] A.K. Nguyen, H.J. Skadsem, A. Brataas, Phys. Rev. Lett. 98 (2007) 146602.

[37] X. Jiang, L. Gao, J.Z. Sun, S.S.P. Parkin, Phys. Rev. Lett. 97 (2006) 217202.

[38] O. Eriksson, L. Nordström, A. Pohl, L. Severin, A.M. Boring, B. Johansson, Phys. Rev. B 41 (1990) 11807.

[39] H. Eschrig, M. Sargolzaei, K. Koepernik, M. Richter, Europhys. Lett. 72 (2005) 611.

[40] H. Ebert, M. Battocletti, E.K.U. Gross, Europhys. Lett. 40 (1997) 545.

[41] A.H. MacDonald, S.H. Vosko, J. Phys. C: Solid State Phys. 12 (1979) 2977.

[42] A.S. Núñez, R.A. Duine, Paul Haney, A.H. MacDonald, Phys. Rev. B 73 (2006) 214426.

[43] R.A. Duine, P.M. Haney, A.S. Núñez, A.H. MacDonald, Phys. Rev. B 75 (2007) 014433.

[44] P.M. Haney, D. Waldron, R.A. Duine, A.S. Núñez, H. Guo, A.H. MacDonald, Phys. Rev. B 75 (2007) 174428.

[45] P.M. Haney, A.H. MacDonald, arXiv:0708.3231v1, 2007.

[46] Z. Wei, A. Sharma, A.S. Núnez, P.M. Haney, R.A. Duine, J. Bass, A.H. MacDonald, M. Tsoi, Phys. Rev. Lett. 98 (2007) 116603.

[47] J. Nogués, I.K. Schuller, J. Magn. Magn. Mater. 192 (1999) 203.

[48] A.E. Berkowitz, K. Takano, J. Magn. Magn. Mater. 200 (1999) 552.

[49] C.L. Fu, A.J. Freeman, Phys. Rev. B 33 (1986) 1755.

[50] S. Urazhdin, N. Anthony, Phys. Rev. Lett. 99 (2007) 046602.

[51] Y. Xu, S. Wang, K. Xia, arXiv:0708.2143, 2007.

[52] O.G. Shpyrko, E.D. Isaacs, J.M. Logan, Y. Feng, G. Aeppli, R. Jaramillo, H.C. Kim, T.F. Rosenbaum, P. Zschack, M. Sprung, S. Narayanan, A.R. Sandy, Nature 447 (2007) 68.

[53] J.R. Petta, S.K. Slater, D.C. Ralph, Phys. Rev. Lett. 93 (2004) 136601.

[54] K. Tsukagoshi, B.W. Alphenaar, H. Ago, Nature 401 (1999) 572.

[55] D. Waldron, P. Haney, B. Larade, A. MacDonald, H. Guo, Phys. Rev. Lett. 96 (2006) 166804.

[56] M. Misiorny, Józef Barnás, arXiv:0706.2315, 2007.

[57] J. Fransson, arXiv:0707.2600v1, 2007.

[58] A. Nitzan, M.A. Ratner, Science 300 (2003) 1384.

[59] F. Evers, F. Weigend, M. Koentopp, Phys. Rev. B 69 (2004) 235411.

[60] N. Sai, M. Zwolak, G. Vignale, M. Di Ventra, Phys. Rev. Lett. 94 (2005) 186810.

[61] J.P. Eisenstein, A.H. MacDonald, Nature 432 (2004) 691.

[62] E. Rossi, A.S. Núñez, A.H. MacDonald, Phys. Rev. Lett. 95 (2005) 266804. 